\title{
ZIP7 (SLC39A7) expression in colorectal cancer and its correlation with clinical prognosis
}

\author{
Yang Luo ${ }^{1 \#}$, Yicheng Shen ${ }^{1 \#}$, Zhong Ju' ${ }^{2}$ Zhi Zhang ${ }^{1}$ \\ ${ }^{1}$ Department of General Surgery, Suzhou Ninth People's Hospital, Suzhou, China; ${ }^{2}$ Department of Dermatology, Suzhou Municipal Hospital, \\ Suzhou, China \\ Contributions: (I) Conception and design: Y Luo; (II) Administrative support: Y Shen; (III) Provision of study materials or patients: Z Zhang; (IV) \\ Collection and assembly of data: Z Ju; (V) Data analysis and interpretation: Y Luo; (VI) Manuscript writing: All authors; (VII) Final approval of \\ manuscript: All authors. \\ \#These authors contributed equally to this work. \\ Correspondence to: Yicheng Shen. Department of General Surgery, Suzhou Ninth People's Hospital, Suzhou 215000, China. \\ Email: shenyicheng2019@163.com.
}

Background: Colorectal cancer (CRC) is a common gastrointestinal malignant tumor that occurs in the colon site and accounts for $9 \%$ of the total malignant tumors. Among malignant tumors, its morbidity and mortality respectively rank third and fourth, seriously threatening human health and causing a heavy economic burden on society. ZIP7 (SLC39A7), a kind of zinc transporter, plays a crucial role in the selfrenewal of intestinal epithelial cells; however, its role in CRC has not been extensively examined. Therefore, our study aimed to analyze the biological function and expression of this zinc transporter in CRC, along with its correlation with disease activity.

Methods: In this study, 118 cases of colorectal carcinoma tissues, 30 normal tissue samples from adjacent cancer tissues, and 30 normal intestinal mucosa tissue samples from non-intestinal cancer patients were collected in our hospital between February 2014 and February 2015. The expressions of ZIP7 were examined immunohistochemically, and the relationship between ZIP7 expression and the clinical pathological features of CRC were analyzed. After appropriate surgical treatment, the patients accepted a 5-year follow-up for evaluation of their recurrence situation and 5-year survival rate.

Results: Immunohistochemically, out of 80 CRC tissue samples, $67.8 \%$ were found to be positive for ZIP7, 55\% (44 cases) were strong positives, and 45\% (36 cases) were weak positives. There was a striking coherence between the expression of ZIP7, the depth of lymph node metastasis, CRC invasion, and CRC Dukes stage $(\mathrm{P}<0.05)$. Subsequent studies indicated that the mortality rate was positively correlated with the staining intensity of ZIP7 $(\mathrm{P}<0.05)$, and the Cox proportional hazards model confirmed that ZIP7 is an independent element of prognostic in patients with CRC (RR =3.896; 95\% CI: 1.526-9.951; $\mathrm{P}=0.004)$.

Conclusions: The high expression of ZIP 7 in epithelial cells is related to the clinical stage and prognosis of CRC and can be utilized as a biomarker to predict the prognosis of CRC patients.

Keywords: ZIP7; gene expression; colorectal neoplasms; prognosis

Submitted Jul 08, 2020. Accepted for publication Sep 18, 2020.

doi: $10.21037 /$ tcr-20-2640

View this article at: http://dx.doi.org/10.21037/tcr-20-2640

\section{Introduction}

Colorectal cancer (CRC) is a common gastrointestinal malignant tumor that occurs in the colon site and accounts for $9 \%$ of the total malignant tumors (1). It is a serious threat to people's health and a heavy economic burden for the public (2). Although there is no specific reason for the occurrence of CRC, studies have shown that many factors are closely related to its occurrence, such as the degree of 
economic development, lifestyle, gender, and age $(3,4)$. In addition, previous history of colon polyps, inflammatory bowel disease (such as ulcerative colitis or Crohn's disease) and genetic factors are also associated with the incidence of CRC. The development of CRC involves different molecules and signaling pathways, which can influence each other and interact with further pathways to accelerate tumor formation and progression (5).

According to data from the International Agency for Research on Cancer (IARC), nearly 1 million new cases of colon cancer were expected in 2018, and more than 500,000 deaths. Moreover, its incidence and mortality rate are ranked fifth in China (6). For patients with CRC, surgery is undoubtedly the only cure that can be cured. However, with the development of radiotherapy, chemotherapy and targeted therapy, multidisciplinary treatment (MDT) has been able to greatly improve the survival time and quality of life of CRC patients. The current NCCN and ASCO guidelines both recommend preoperative neoadjuvant treatment for patients with T3 and T4 CRC, which can help reduce tumors, so as to reduce the difficulty of surgery and increase the patient's anus preservation rate. The current intensity-modulated radiotherapy and spiral tomography radiotherapy system TOMO, combined with the CAPOX program can significantly increase the $\mathrm{pCR}$ rate of CRC patients. But for patients with liver metastases from CRC, the main treatment is liver resection, but more than $30 \%$ of patients will relapse within one year after partial hepatectomy (7-9). The 5-year relative survival rate of patients with stage I disease is higher than $90 \%$, while the 5-year relative survival rate of patients with stage IV disease is only slightly higher than $10 \%(10)$. Thus, to understand and treat CRC development, the molecular mechanism behind its progression needs to be clarified, while any biomarkers and prospective therapeutic targets require urgent identification.

Zinc is an essential trace element in the human body, a component in various metal enzymes, and an enzyme activator. It is integral to the development of the body, the immune mechanism, and the metabolism of various proteins and nucleic acids. The homeostasis of zinc ion metabolism acts as an essential part in maintaining the absorption, storage, and loss of zinc ions (11).

Previous studies have revealed that the zinc ion transporter family, SLC39A (ZIP) is directly involved in the steady state of zinc ions in the cell, it increases the intracellular $\mathrm{Zn} 2+$ concentration by uptake of extracellular $\mathrm{Zn} 2+$ or release of $\mathrm{Zn} 2+$ in intracellular vesicles. The
SLC39A transport family exhibits unique tissue-specific expression and exhibits specific responses to physiological stimuli through hormones and cytokines. The human SLC39 gene family has at least 14 members and is divided into 4 subfamilies: I, II, GufA, and LIV-1. Subfamily type I includes ZIP9; subfamily type II includes ZIP1, ZIP2, ZIP3; subfamily GufA type includes ZIP11; subfamily LIV-1 includes ZIP4-8, ZIP10, and ZIP12-14. ZIP family proteins are also related to hormones, and also has an important impact on the occurrence and progress of tumors and other diseases. Normal concentration of zinc can inhibit the activation of nuclear factor kappa-light-chain-enhancer of activated B cells (NF-KB), heighten the body's sensitivity to TNF- $\alpha$, and induce an antitumor effect (12). Studies have found that ZIP4 expression is elevated in human pancreatic cancer and may accelerate pancreatic cancer metastasis through tumor progression (13). ZIP6 is located on the cytoplasmic membrane, which will lead to increased intracellular zinc levels. Currently, ZIP6 has been found to be abnormally expressed in digestive system tumors (such as esophageal cancer, liver cancer, and pancreatic cancer), indicating that the protein may have a critical role in tumorigenesis (14). ZIP10 participate in the occurrence and metastasis of breast cancer by promoting cell proliferation and enhancing metastatic ability $(15,16)$. Furthermore, screening of the entire gene expression profile of esophageal cancer tissue revealed abnormal expression of 19 genes including ZIP5, indicating that ZIP5 may be involved in esophageal cancer (17).

SLC39A7 (ZIP7) is a zinc transporter located on the endoplasmic reticulum and the Golgi. In breast cancer cells, activated ZIP7 can in turn activate cell growth and proliferation-related signaling access, including that of MAPK, PI3K, and mTOR (18). It was also notably found to be upregulated in a gastric tumor model (19) and is considered to play an essential role in the self-renewal of epithelial cells (20). ZIP7 is also crucial to the survival and proliferation of CRC cells, role, and is thus relevant to the development of CRC tumors, Knockdown of ZIP7 will greatly reduce the viability and proliferation of colon cancer cells. It was also shown that knockdown of ZIP7 interfered with cell cycle progression and induced G2/M cell cycle arrest and enhanced early and late apoptosis of CRC cells. In addition, the down-regulation of ZIP7 promoted the cleavage of PARP, enhanced the expression of Bad, Caspase- 9 and Caspase-3, and inhibited the expression of Bcl-2 (21).

Despite this research, the clinical relationship between 
ZIP7 and CRC has never been extensively investigated. Therefore, this study aimed to explore the relationship between ZIP7 expression in CRC tissue and its relationship with CRC invasion, metastasis, and clinical staging. To further analyze ZIP7's clinical significance and to initially evaluate the relationship between ZIP7 expression and clinical efficacy, patients were followed up during intervention. It is hoped the information gleaned from this study will provide more comprehensive insights into the occurrence, development, and prognosis of CRC. We present the following article in accordance with the REMARK reporting checklist (available at http://dx.doi. org/10.21037/tcr-20-2640).

\section{Methods}

\section{Setting and study design}

From February 2014 to February 2015, 118 consecutive CRC patients were enrolled in Suzhou Ninth People's Hospital. Among them, 68 were male and 50 were female, aged from $26-81$, with an average age of $(56.3 \pm 17.2)$ years. The inclusion criteria were the following: (I) undergoing radical CRC surgical treatment; (II) postoperative pathology confirmed as primary CRC; (III) receiving postoperative radiotherapy and chemotherapy; (IV) complete clinical and pathological data; (V) patient signing informed consent. Meanwhile, the exclusion criteria were the following: (I) relapsed CRC; (II) combined with other types of primary malignant tumors; (III) combined with severe blood system disease, immune system disease, endocrine system disease, cardiovascular disease or cerebrovascular disease, or other organ and tissue disease of the liver, kidney, lung etc.; (IV) serious complications or death occur within 30 days after surgery. This study was conducted in accordance with the ethical principles of the Declaration of Helsinki, and has been approved by the ethics committee of the Suzhou Ninth People's Hospital (No. 20140013). Written informed consent was obtained from the patient.

According to the specific situation of the patient and after the corresponding treatment, the patient's cancer tissue section was preserved, and the clinical and pathological data (Dukes stage, lymph node metastasis, and invasion depth) were recorded in detail for subsequent analysis. In addition, 30 normal tissue samples from adjacent cancer tissues and 30 normal intestinal mucosa tissue samples from nonintestinal cancer patients were collected as controls. The specimens of medical examiners who came to our hospital for colonoscopy from February 2014 to February 2015 were randomly selected as tissue specimens of non-intestinal cancer patients. All the included patients participated in the follow-up survey after receiving the corresponding treatment, 3 of whom were lost to follow-up; 24 patients died after 1 year of follow-up, 40 patients died after 3 years of follow-up, and 47 died after 5 years of follow-up. Among these, 46 died of tumor metastasis and recurrence, and 1 died of other diseases.

\section{Immunobistochemistry}

All precipitates of tissue samples for immunohistochemical determination were fixed with formalin, embedded in paraffin, then sectioned, and mounted on charged slides. The primary CRC sample was washed $(\mathrm{n}=118)$ with different concentrations of ethanol (70-100\%) and xylene to rehydrate the sample. Various antigen recovery conditions were tested to determine the best condition for pZIP7 staining by cooking the microwave in a buffer of $\mathrm{pH}$ 8 EDTA acid at $950 \mathrm{~W}$ for 2 minutes. The determination used the mouse monoclonal pZIP7 antibody (MABS1262, EMD Millipore, USA) optimized to a $1 / 8,000$ dilution for the cell pellets and 1/800 dilution for colon cancer sections. The sample was washed with $0.02 \%$ phosphate-buffered saline (PBS)/Tween, and then the sample was covered with $0.18 \%$ hydrogen peroxide solution to block endogenous peroxidase. Next, the sections were blocked with serum-free blocking agent (Dako, UK) for 20 minutes.

The pZIP7 antibody was incubated for 1 hour at room temperature in a humidity cabinet with the optimal dilution of pellet to cells, and the tissue sample was placed in a $23{ }^{\circ} \mathrm{C}$ environment for 12 hours. The slides were then washed twice with $0.02 \% \mathrm{PBS} /$ Tween, detected with mouse envision polymer-hpr k4001 (Dako, UK) for 1 hour, and then washed twice with $0.05 \%$ TBS/tween. Next, 3'-3'-diaminobenzidine (DAB) chromogen substrate solution (Dako, UK) was used to observe the target protein.

Use $0.05 \%$ methyl green (aqueous solution) for counterstaining. Then use the DPX cover glass and observe on the Olympus BH-2 microscope. After determining the representative assessment area, the ZIP7 immunostaining of the sample was assessed by two people, and the sample was divided into negative [0], weak [1], and strong staining [2] according to the staining intensity category, and the positive rate was recorded. The score was calculated according to the above calculation method and recorded. 
Table 1 ZIP7 protein expression n (\%)

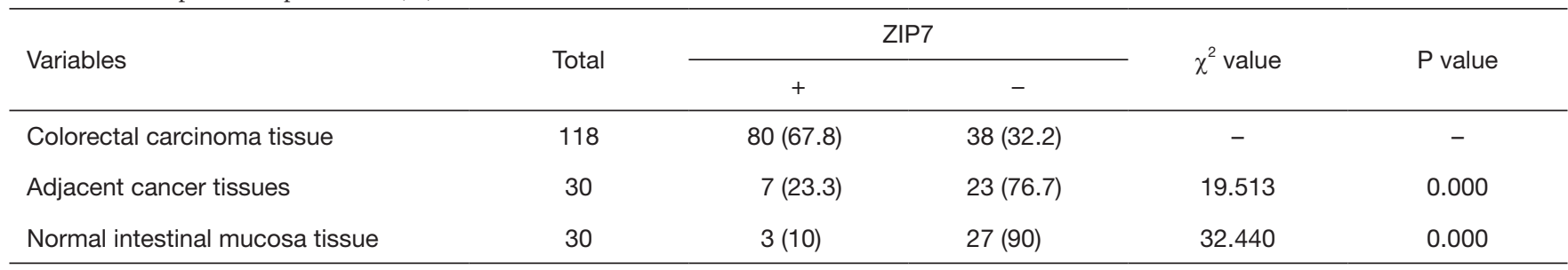
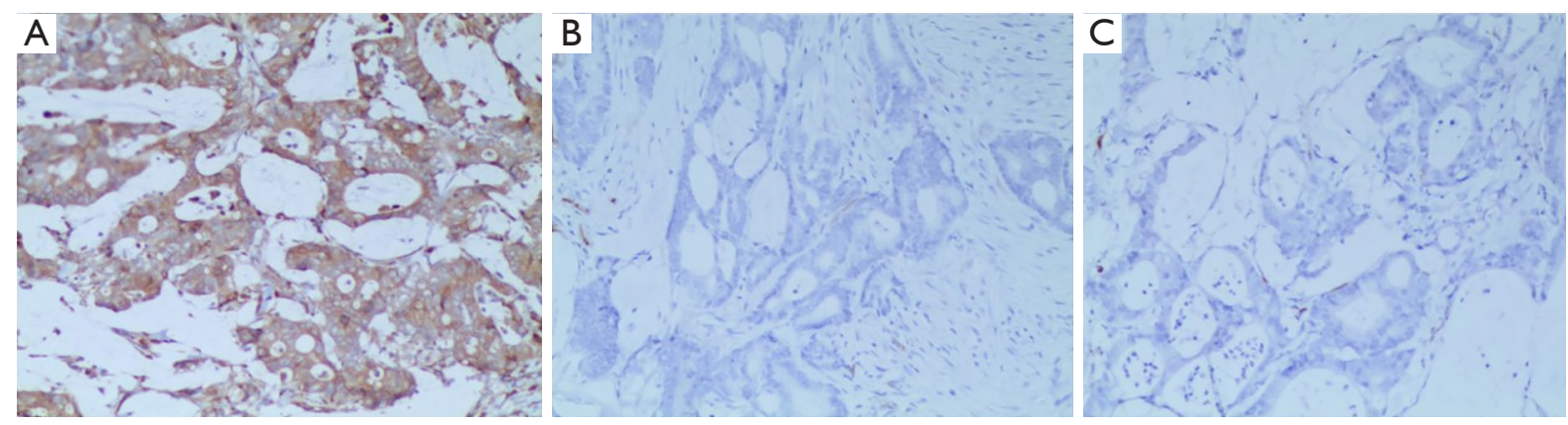

Figure 1 Expression of ZIP7 in CRC tissues/adjacent tissues/and normal intestinal mucosa tissues: (A) CRC tissue, (B) adjacent tissues, (C) normal intestinal mucosa tissues. Scale bars $=50 \mu \mathrm{m}$. CRC, colorectal cancer.

\section{Fudgment of results}

Scoring was determined according to the number of positive cells and the staining intensity. Five 200-fold visual fields were randomly selected for each section to be used for counting of the number of positive cells and the determination of staining intensity. According to the relevant guidelines of the American Society of Clinical Oncology (ASCO), the ZIP7 expression intensity is divided into 3 levels: (I) scoring standard for staining intensity, 0 points $=$ no staining, 1 point $=$ light staining (slightly stronger than negative control), 2 points = severe staining; (II) scoring for the number of positive cells, 1 point $\leq 30 \%$ cell staining, 2 points $\geq 30 \%$ cell staining. All specimens were jointly diagnosed by two senior pathologists.

\section{Postoperative follow-up}

All patients were followed up by telephone or outpatient follow-up, with the day of diagnosis as the starting point for follow-up, and March 31, 2020 as the deadline for follow-up.

\section{Statistical analysis}

Continuous variables are reported as mean with standard deviation, and other categorical variables are reported as numbers with percentages. Accordingly, the differences of continuous variables and categorical variables between two groups were analyzed using the $\chi^{2}$ test. Cox proportional hazards model was used to analyze the risk factors of overall survival in CRC patients. When $\mathrm{P}<0.05$, the difference was considered to be significant. Statistical analysis was performed using Stata 22.0.

\section{Results}

\section{Upregulation of ZIP7 in buman CRC}

Immunohistochemical staining results showed that colored particles were visible in the cell membrane and/or cytoplasm of CRC tissues, adjacent tissues, and normal intestinal mucosa tissues, indicating that ZIP7 is expressed in CRC tissues, adjacent tissues, and normal intestinal mucosa tissues. The positive expression rates of ZIP7 in CRC tissues, adjacent tissues, and normal intestinal mucosa tissues were $67.8 \%, 23.3 \%$, and $10 \%$, respectively, and the differences were statistically significant $(\mathrm{P}<0.05)$ (Figure 1, Table 1).

\section{Relationship between ZIP7 and the clinical features of CRC}

The differences of ZIP7 expression between Dukes stage, 
Table 2 Relationship between ZIP7 protein expression and clinicopathological characteristics of patients with colorectal cancer n (\%)

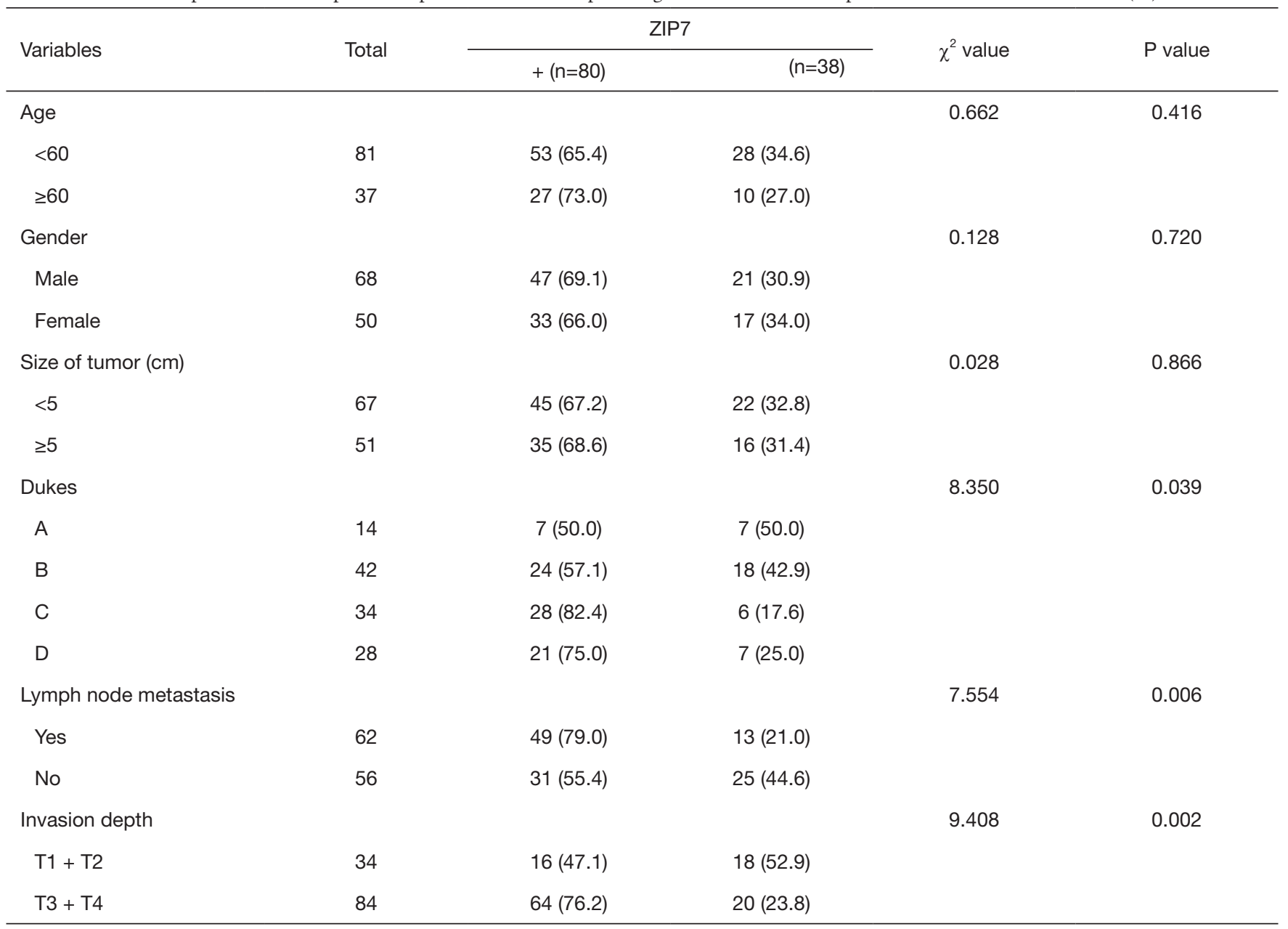

lymph node metastasis, and invasion depth were statistically significant $(\mathrm{P}<0.05)$, while the differences between gender, age, and tumor size were not statistically significant $(\mathrm{P}>0.05)$ (Table 2).

\section{Relationship between ZIP7 and the prognosis of colon cancer}

With the mean value of ZIP7 relative expression level as the boundary, positive staining for ZIP7 was found in 80 CRC tissue samples $(67.8 \%)$, of which 44 (55\%) were strongly positive and $36(45 \%)$ were weakly positive. As of the follow-up deadline, a total of 47 patients died and 3 patients were lost to follow-up. The follow-up rate was $97.46 \%(115 / 118)$. Of the 115 patients included in the analysis, 43 had a high expression of ZIP7, 35 had a low expression, and 37 had a negative expression. The mortality rates of the three groups during the follow-up period were $69.8 \%$ (30/43), $28.6 \%(10 / 35)$, and $13.5 \%$ (5/37), respectively. ZIP7 high expression in CRC tissue samples was significantly higher than low expression and negative expression, while low expression was significantly higher than negative expression $(\mathrm{P}<0.05)$. Cox multivariate analysis showed that the depth of tumor invasion, lymph node metastasis, Dukes stage, and ZIP7 were independent factors affecting the prognosis of patients $(\mathrm{P}<0.05)$ (Figure 2, Tables 3 and 4).

\section{Discussion}

An important biological characteristic of malignant tumors is their extremely strong ability for invasion and migration. This ability is not only related to the clinical treatment effect and prognosis of patients, but also the main reason 
for patients' postoperative recurrence and survival rate decline. CRC is one of the most common malignant tumors throughout world, and its poor survival rate is mainly due to the late diagnosis of this aggressive disease and the limited effectiveness of currently available treatment strategies $(22,23)$. Therefore, understanding the molecular mechanism of CRC comprehensively can help realize the early diagnosis and treatment of CRC, which in turn may improve the 5-year survival rate of CRC after surgery.

SLC39A7 can secrete zinc from the endoplasmic reticulum and Golgi apparatus to the cytoplasm, increase the concentration of zinc ions in the cytoplasm, and redistribute zinc from the intracellular compartment to the cytoplasm through ZIP7, which can cause the activation of growth factor receptors and zinc-induced phosphatase. Inhibition,

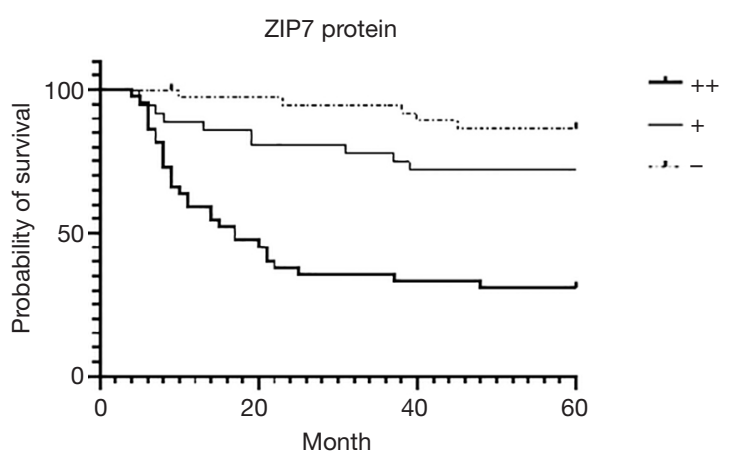

Figure 2 Relationship between ZIP7 and the prognosis of color cancer. thereby preventing the dephosphorylation of tyrosine kinase receptors, which are often abnormally expressed and activated in cancer (24). ZIP7 and zinc were found to be increased in tamoxifen-resistant breast cancer MCF-7 cells, which exhibit an invasive and aggressive phenotype through zinc-induced activation of EGFR, IGF-1R receptor tyrosine kinase, and non-receptor tyrosine Src kinase (25). ZIP7 has been proven to have multiple stimulatory functions that can trigger apoptotic cell death. In higher eukaryotes, there are two main types of apoptosis pathways, namely deathreceptor pathway and the mitochondrial pathway (26). In breast cancer cells, activated ZIP7 can activate cell growth and proliferation-related signaling access, such as MAPK, PI3K, and mTOR signaling access (18).

This study examined the expression of ZIP7 in CRC through a comparative study of 118 pathological specimens of CRC patients, 30 precancerous tissue specimens, and 30 normal bowel mucosa tissue specimens of non-intestinal cancer patients. The results of staining indicated that the expression of ZIP7 in the pathological tissues of the CRC patients was higher than that of adjacent tissues and normal intestinal mucosa tissues, which is consistent with the results of Ohashi et al. (20), further confirming that ZIP7 is highly expressed in tumors and is closely relevant to tumor development. These findings cement the assumption that the expression of ZIP7 is closely related to the occurrence of tumors. Statistical analysis of the clinical and pathological data of the patients indicated that the expression of ZIP7 was relevant to the Dukes stage of CRC. In addition, this study also analyzed the relationship between the patients'

Table 3 Multifactor analysis of prognostic factors of colorectal cancer (CRC) patients (Cox proportional hazards model)

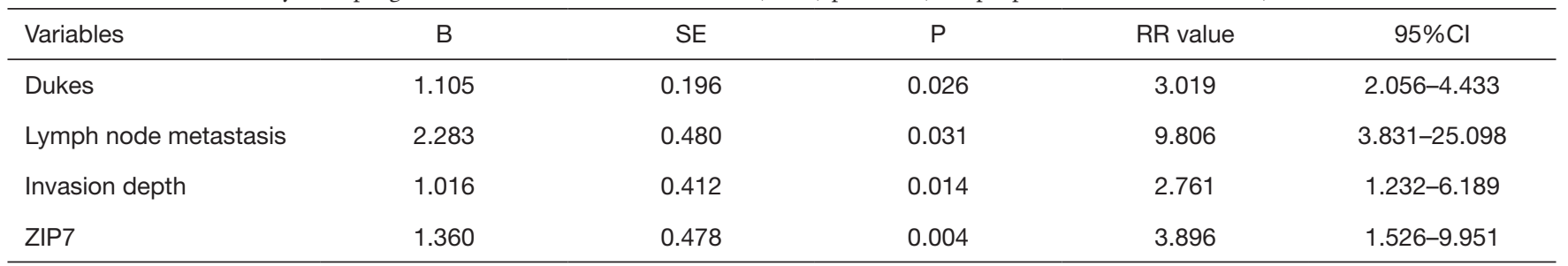

Table 4 Relationship between postoperative recurrence of colorectal cancer and expression level of ZIP7 n (\%)

\begin{tabular}{lcccc}
\hline \multirow{2}{*}{ Recurrence } & Total & \multicolumn{2}{c}{ ZIP7 } & $\chi^{2}$ value \\
\cline { 3 - 4 } & & $40(88.9)$ & $5(11.1)$ & 15.021 \\
Yes & 45 & $38(54.3)$ & $32(45.7)$ & 0.000 \\
No & 70 & P value & \\
\hline
\end{tabular}


5 -year survival and postoperative recurrence and ZIP7. The results showed that the recurrence rate of the patients in the ZIP7-positive expression group was relatively higher than that in the negative one, and the 5-year postoperative survival time was significantly shortened, which was not conducive to the prognosis of patients. The expression of ZIP7 thus has important reference significance for judging the pathological process and stage of CRC and has potential as a new molecular marker to measure the pathological process and metastasis of CRC.

In conclusion, ZIP7 is highly expressed in CRC tissues, and is closely relevant to cancer progression, metastasis, and prognosis of patients. Therefore, the individual monitoring of ZIP7 in CRC may be critical to understanding the biological characteristics of tumors and ascertaining individual prognosis.

\section{Acknowledgments}

Funding: None.

\section{Footnote}

Reporting Checklist: The authors have completed the REMARK reporting checklist. Available at http://dx.doi. org/10.21037/tcr-20-2640

Data Sharing Statement: Available at http://dx.doi. org/10.21037/tcr-20-2640

Conflicts of Interest: All authors have completed the ICMJE uniform disclosure form (Available at http://dx.doi. org/10.21037/tcr-20-2640). The authors have no conflicts of interest to declare.

Ethical Statement: The authors are accountable for all aspects of the work in ensuring that questions related to the accuracy or integrity of any part of the work are appropriately investigated and resolved. This study was conducted in accordance with the ethical principles of the Declaration of Helsinki (as revised in 2013), and has been approved by the ethics committee of the Suzhou Ninth People's Hospital (No. 20140013). Written informed consent was obtained from the patient.

Open Access Statement: This is an Open Access article distributed in accordance with the Creative Commons Attribution-NonCommercial-NoDerivs 4.0 International
License (CC BY-NC-ND 4.0), which permits the noncommercial replication and distribution of the article with the strict proviso that no changes or edits are made and the original work is properly cited (including links to both the formal publication through the relevant DOI and the license). See: https://creativecommons.org/licenses/by-nc-nd/4.0/.

\section{References}

1. Siegel RL, Miller KD, Jemal A. Cancer statistics, 2020. CA Cancer J Clin 2020;70:7-30.

2. Islami F, Goding Sauer A, Miller KD, et al. Proportion and number of cancer cases and deaths attributable to potentially modifiable risk factors in the United States. CA Cancer J Clin 2018;68:31-54.

3. Yang Y, You YN. Comprehensive clinical genetics care for patients with inherited colorectal cancer associated with Lynch syndrome: Western and Asian perspectives. Chin Clin Oncol 2018;7:9.

4. Freedman AN, Slattery ML, Ballard-Barbash R, et al. Colorectal cancer risk prediction tool for white men and women without known susceptibility. J Clin Oncol 2009;27:686-93.

5. Yan Z, Wang R, Su Q. A novel cancer stem cell-based classification model for the tumorigenesis and development of colorectal cancer. Transl Cancer Res 2019;8:2621-3.

6. Fang Y, Xiao B, Peng J, et al. An early report of a screening program for colorectal cancer in Guangzhou, China. Ann Transl Med 2019;7:604.

7. Blazer DG 3rd, Kishi Y, Maru D M, et al. Pathologic response to preoperative chemotherapy: a new outcome end point after resection of hepatic colorectal metastases. J Clin Oncol 2008;26:5344-51.

8. Vauthey JN, Zimmitti G, Kopetz SE, et al. RAS mutation status predicts survival and patterns of recurrence in patients undergoing hepatectomy for colorectal liver metastases. Ann Surg 2013;258:619-26; discussion 626-7.

9. Rubbia-Brandt L, Giostra E, Brezault C, et al. Importance of histological tumor response assessment in predicting the outcome in patients with colorectal liver metastases treated with neo-adjuvant chemotherapy followed by liver surgery. Ann Oncol 2007;18:299-304.

10. Howlader N, Noone AM, Krapcho M, et al. SEER Cancer Statistics Review,1975-2016. National Cancer Institute; 2019.

11. Lichten LA, Cousins JA, Mammalian. Zinc transporters: nutritional and physiologic regulation. Annu Rev Nutr 2009;29:153-76. 
12. Uzzo R G, Crispen P L, Golovine K, et al. Diverse effects of zinc on NF-kB and AP-1 transcription factors: implications for prostate cancer progression. Carcinogenesis 2006;27:1980-90.

13. Li M, Zhang Y, Liu Z, et al. Aberrant expression of zinc transporter ZIP4 (SLC39A4) significantly contributes to human pancreatic cancer pathogenesis and progression. Proc Natl Acad Sci USA 2007;104:18636-41.

14. Taylor KM. A distinct role in breast cancer for two LIV-1 family zinc transporters. Biochem Soc Trans 2008;36:1247-51.

15. Taylor KM, Vichova P, Jordan N, et al. ZIP7-mediated intracellular zinc transport contributes to aberrant growth factor signaling in antihormone-resistant breast cancer cells. Endocrinology 2008;149:4912-20.

16. Kagara N, Tanaka N, Noguchi S, et al. Zinc and its transporter ZIP10 are involved in invasive behavior of breast cancer cells. Cancer Sci 2007;98:692-97.

17. Anupam K, Tusharkant C. Discovery of deregulation of Zinc homeostasis and its associated genes in esophageal squamous cell carcinoma using cDNA microarray. Int J Cancer 2007;120:230-42.

18. Nimmanon T, Ziliotto S, Morris S, et al. Phosphorylation of zinc channel ZIP7 drives MAPK, PI3K and mTOR growth and proliferation signalling. Metallomics 2017;9:471-81.

19. Itadani H, Oshima H, Oshima M, et al. Mouse gastric tumor models with prostaglandin E2 pathway activation

Cite this article as: Luo Y, Shen Y, Ju Z, Zhang Z. ZIP7 (SLC39A7) expression in colorectal cancer and its correlation with clinical prognosis. Transl Cancer Res 2020;9(10):6471-6478. doi: $10.21037 /$ tcr-20-2640 show similar gene expression profiles to intestinal-type human gastric cancer. BMC Genomics 2009;10:615.

20. Ohashi W, Kimura S, Iwanaga T, et al. Zinc Transporter SLC39A7/ZIP7 Promotes Intestinal Epithelial SelfRenewal by Resolving ER Stress. PLoS Genet 2016;12:e1006349.

21. Sheng N, Yan L, You W, et al. Knockdown of SLC39A7 inhibits cell growth and induces apoptosis in human colorectal cancer cells. Acta Biochim Biophys Sin (Shanghai) 2017;49:926-34.

22. Siegel RL, Miller KD, Fedewa SA, et al. Colorectal cancer statistics, 2017. CA Cancer J Clin 2017;67:177-93.

23. Siegel RL, Miller KD, Jemal A. Cancer Statistics, 2017. CA Cancer J Clin 2017;67:7-30.

24. Hogstrand C, Kille P, Nicholson RI, et al. Zinc transporters and cancer: a potential role for ZIP7 as a hub for tyrosine kinase activation. Trends Mol Med 2009;15:101-11.

25. Taylor KM, Vichova P, Jordan N, et al. ZIP7-mediated intracellular zinc transport contributes to aberrant growth factor signaling in antihormone-resistant breast cancer Cells. Endocrinology 2008;149:4912-20.

26. Gupta S. Molecular steps of death receptor and mitochondrial pathways of apoptosis. Life Sci 2001;69:2957-64.

(English Language Editor: J. Gray) 